\title{
Fractional-Order-Based ACC/CACC Algorithm for Improving String Stability
}

\author{
Carlos Flores and Vicente Milanés.
}

Carlos Flores is with the Robotics and Intelligent Transportation Systems (RITS) Team, INRIA Paris, 2 Rue Simone Iff, 75012 France

Vicente Milanés is with the Research Department, Renault SAS, 1 Avenue de Golf, 78280 Guyancourt, France

\begin{abstract}
Traffic flow optimization and driver comfort enhancement are the main contributions of an Adaptive Cruise Control (ACC) system. If communication links are added, more safety and shorter gaps can be reached performing a Cooperative-ACC (CACC). Although shortening the inter-vehicular distances directly improves traffic flow, it can cause string unstable behavior. This paper presents fractional-order-based control algorithms to enhance the car-following and string stability performance for both ACC and CACC vehicle strings, including communication temporal delay effects. The proposed controller is compared with state-of-the-art implementations, exhibiting better performance. Simulation and real experiments have been conducted for validating the approach.
\end{abstract}

Keywords: Adaptive Cruise Control, Cooperative Adaptive Cruise Control, String Stability, Fractional-Order Calculus, Cooperative Systems, Intelligent Transportation Systems

10

\section{Introduction}

Car-following systems are of the most promising Advanced Driver Assistance System (ADAS) for improving both traffic safety and flow. It is based on a front range sensor for detecting the preceding vehicle, adapting the ego-vehicle speed accordingly-i.e. Adaptive Cruise Control (ACC). This technique permits to efficiently track the preceding vehicle maintaining a desired distance. The evolution of such already 15 commercial technology is the Cooperative-ACC (CACC), which proposes to add vehicle-to-vehicle (V2V) communication links to maintain tigher string formations.

Recent works have demonstrated CACC benefits with respect to ACC 19. The main contributions of CACC systems are an improvement in traffic throughput due to shorter inter-vehicle distances and the enhancement of drivers' safety and comfort 38. As main limitations, the V2V communications must be guaranteed for achieving these results. Moreover, the temporal delay that these links may present affects the dynamic performance of the car-following maneuver, threatening directly the string stability and leading to undesirable behavior.

Platooning systems have been firstly tested on real roads by the Partners for Advanced Transportation Technology (PATH) in 1997 34, performing platoon formations with highly short inter-distances, which required high interaction with the leader vehicle and dedicated lanes. More recently, the PATH has also contributed with several research works in CACC [25, 39, 37. Other projects as Connect \& Drive [6] have approached CACC considering possible interaction with other cars in highways. They have shown positive results that demonstrate an increase in terms of safety and traffic throughput. In addition, this technique has gained more attention recently through research competitions as the first Grand Cooperative

\footnotetext{
${ }^{1}$ http://www.gcdc.net/en/i-game 
The platoon frequency response and the string stability notion are addressed by the work of [23]. Other approaches have applied different control structures and techniques to provide encouraging performances; such as Model Predictive Control (MPC) [14, sliding mode control [15] or $\mathcal{H}_{\infty}$ controller-based approaches [31, 8]. Robust performances have been obtained when more complex topologies are used [48, 35]. Finally, 35 one-vehicle look-ahead feedforward structures with Proportional-Derivative (PD) feedback controllers [20, 30 have been widely employed in the literature providing great results due to its damping properties, practical implementation and low computational cost.

Almost all state-of-the-art approaches that address this problem are based in integer order control (IOC), which encourages the usage of non-integer order techniques to reach even more exigent performances. Frac-

40 tional order control has arisen as a frequently employed mathematical tool to fulfil more demanding design requirements, due to its capabilities to provide a more precise and adaptable frequency response [46. This technique has been already employed in car-following approaches, firstly for a hybrid ACC control design [10], a low level throttle and brake control for ACC [11] and finally a CACC controller robust against plant gain disturbances [7].

This work proposes to employ fractional control to design feedforward structures for both ACC and CACC, aiming to enhance the stability and achieve formations with shorter time gaps and improved system performance. It is proposed to profit from the more adaptable frequency response that fractional-order control (FOC) provides in comparison to classical controllers. The performance is studied through string stability analysis of the designed controller and state-of-the-art solutions for ACC and CACC. The improve-

50 ments in terms of string stability of the FOC approach with respect to state-of-the-art IOC solutions are demonstrated in simulations and real platforms tests.

The rest of this paper is structured as follows: Section II introduces the concepts and definitions associated to ACC/CACC systems. An introduction to fractional-order calculus and its applications in control is stated in Section III. Section IV describes the model of the experimental platform that is going to be

55 used for the controller synthesis. The proposed feedforward structure and the controller design procedure for ACC and CACC are presented in Section V and VI respectively. In Section VII, a comparative study of the proposed algorithm for both ACC and CACC systems is described, including a comparison with state-of-the-art developments. The conclusions derived from the obtained results and possible future work are stated in the Section VII.

\section{Concept and definitions}

When it comes to analyze car-following string of vehicles, there are two main concepts to be considered: 1) the adopted car-following policy; and 2) the string stability of the formed platoon. This section reviews both concepts.

Attending to the car-following policy, different strategies can be found in the literature:

1. Constant clearance was conceived as a strategy aimed mainly for platooning maneuvers [42, which consists on driving in highway with really close distance gaps. This approach requires dedicated lanes and low latency $\mathrm{V} 2 \mathrm{~V}$ communication links with the leader vehicle to handle very short spacing.

2. Constant Time Gap (CTG) constitutes one of the most employed and flexible spacing policies. It suggests to perform a vehicle tracking with non fixed distances 43. It simulates the way how humans drive since it proposes to set higher distances when the speed increases, using a time gap multiplied by the ego-speed added to a fixed standstill distance.

3. Constant safety factor is another frequently employed spacing policy [12]. This technique proposes to have a more reactive and conservative behavior in case a hard braking is executed by a forward vehicle, adding a term that penalizes the difference between both vehicles' speeds.

4. Variable Time Gap (VTG) is a spacing policy that has been addressed by some recent research works, suggesting to variate the time gap while driving, with different purposes and in function of the ego-speed. For example, accomplishing improved traffic flow stability [49] or enhanced safety [47] and comfort [18]. 
Another fundamental notion and important design requirement of car-following techniques is the string stability, which states that every string formation must not propagate the disturbances upstream. This concept can be understood from the scope of Lyapunov stability, where it may be interpreted as the asymptotic stability of a finite number of interconnected individual exponentially stable systems [41. The main objective of the car-following-oriented approaches that address this phenomenon is to ensure the attenuation of any perturbation along the string, avoiding undesired behaviors. This concept is better described in equations:

$$
\begin{gathered}
\left\|z_{i}(t)\right\|_{\infty} \leq\left\|z_{i-1}(t)\right\|_{\infty} ; \forall t \geq 0 ; 2 \leq i \leq m ; \\
\|\Gamma(j \omega)\|_{\infty} \equiv\left\|\frac{Z_{i}(j \omega)}{Z_{i-1}(j \omega)}\right\|_{\infty} \leq 1 ; \forall \omega>0 ;
\end{gathered}
$$

where $z_{i}(t)$ is understood as a representative dynamic variable of the $i^{\text {th }}$ vehicle of an homogeneous string of $m$ members-i.e. spacing error, position, speed or acceleration-and $Z_{i}(j \omega)$ its frequency domain interpretation. Both Eq. 1 and 2 describe the fact that the ego-vehicle should not amplify the behavior of its preceding car, so the string formation can be extended boundlessly maintaining the desired behavior. The

90 CTG spacing policy is employed in this work, given that it allows to determine the string stability limit of the system directly by surveying the minimal allowed time gap that guarantees a string stable gap-regulation control.

\section{Fractional-Order Calculus}

When it comes to phenomena representation through mathematical modelling, the integer-order calculus has been always the main tool employed in science and engineering applications [1]. However, the necessity of more descriptive and precise solutions yielded that fractional-order control arised as a new tool, gaining more and more attention due to its capability for modelling systems more accurately [2]. Some physical systems are more exactly represented using fractional-order models [44] for example those where the viscoelasticity is fundamental, the notion of fractal robustness [27] or the case of voltage-current relation of a semi-infinite lossy RC line [45. Thus, the generalization of the differential and integral operator of real order results necessary to better describe these phenomena is:

$$
{ }_{a} D_{t}^{\alpha}=\left\{\begin{array}{l}
\frac{d^{\alpha}}{d t^{\alpha}} \rightarrow \alpha \in \mathbb{R}>0 \\
1 \rightarrow \mathbb{R}(\alpha)=0 \\
\int_{a}^{t}(d \tau)^{-\alpha} \rightarrow \alpha \in \mathbb{R}<0
\end{array}\right.
$$

where $D$ is the operator that exist in the time range $t \in(a, t)$ and $\alpha$ the operator order. A more descriptive non-integer order differentiation or integration formula is required to further develop applications with fractional-order calculus. Several time-domain interpretations have been proposed to this operator, where one of the most frequently used was proposed by Caputo 3. It provides a solution where the initial conditions for the fractional differential equation take the same form as for integer-order ones and consequently, they take a physical interpretation. The differentiation operator with respect to time $t$ with a starting point of $t=0$, proposed by Caputo is:

$$
{ }_{0} D_{t}^{\alpha}=\frac{1}{\Gamma(m-\alpha)} \int_{0}^{t} \frac{f^{(m)}(\tau)}{(t-\tau)^{\alpha-m+1}}(d \tau), m-1<\alpha<m, m \in \mathbb{N}
$$

where the term $\Gamma(n)$ is the Euler function. Fig. 1 shows different fractional-order operators applied over a triangular signal with a DC value. It can be appreciated how the signal manipulation changes as the order varies, from the unit gain $(\alpha=0)$ to the first-order derivation $(\alpha=1)$. This operator can be also translated to the frequency domain as:

$$
\mathcal{L}\left\{{ }_{a} D_{t}^{\alpha} f(t)\right\}=s^{\alpha} F(s)
$$




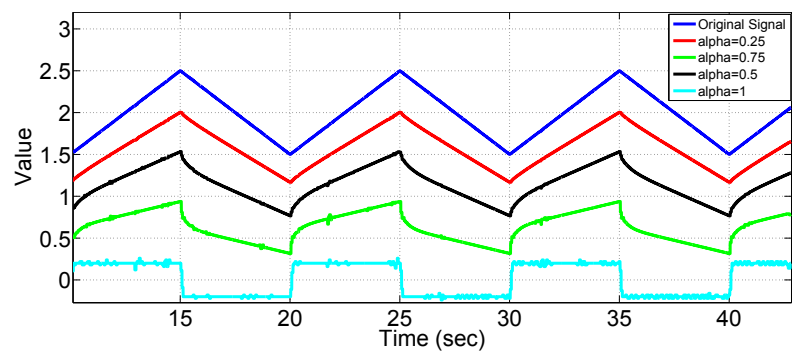

Figure 1: Time plot of a different order derivation applied to a triangular signal

where $f(t)$ and $F(s)$ stands for the function in its time and frequency domain form respectively. As it can be deduced, it constitutes a powerful signal processing feature and consequently, it has been widely employed in the control field in state-of-the-art and industrial approaches with great results [5].

\subsection{Fractional Control}

The integer-order calculus has been the most employed tool for systems control design in industrial environments; being the Proportional-Integral-Derivative (PID) the most employed controller [1. Although IOC has provided great results, the development of more complex control systems requires flexible and accurate controller design methods to satisfy more challenging closed-loop performances. Different control techniques have been proposed that profit from the fractional-order calculus to satisfy more demanding tuning strategies. The first fractional control approach was proposed by [26] with the non-integer robust command (CRONE, from the french Commande Robuste d'ordre non entier) that aims to maintain the system open loop phase margin unvaried, achieving the so-called fractal robustness [27. The fractionalorder lead-lag compensator 21] and the Tilt-Integral-Derivative controller [17] are also other approaches of non-integer controllers that have provided encouraging performances.

In this work, a specific fractional-order control technique is chosen to develop the gap regulation controller, which is the real order generalization of the PID, the $P I^{\lambda} D^{\alpha}$ controller 32. This technique takes as a basis the mathematical form of the PID, stating that its integral and derivative operators $(\lambda$ and $\alpha$ respectively) should not necessarily have order 1 , but it will be possible to have both integration and differentiation of real order. The control law proposed by this approach is described as follows:

$$
C(s)=\frac{E(s)}{U(s)}=K p+K d \cdot s^{\alpha}+K i \cdot s^{-\lambda} ;(\alpha, \lambda)>0
$$

where two design parameters are added $(\alpha, \lambda)$, allowing not only to modify the contribution of each integration and differentiation operator respectively, but also to modify their behavior. $E(s)$ and $U(s)$ represent the spacing error and feedback controller output respectively. This permits to have five design parameters and more flexible frequency response and thus, permitting to move in the plane presented in Fig. 2a instead of only being able to yield control actions within the 4 black points-i.e. P, PD, PI and PID controllers. Another approaches have proposed to employ controllers of the form $P I^{\lambda}\left[22\right.$, or $P D^{\alpha}$ [16, 13] to achieve the desired behavior where it is not required to have all the operators of the real-order PID.

In the frequency domain, by introducing the non-integer orders of the differentiator or integrator, the design process can not only modify the pole/zero placement, but also the whole magnitude and phase contribution of the controller. For example, a differential operator with order $\alpha$ provides a phase increase slope of $+\alpha \frac{\pi}{2} \mathrm{rad} / \mathrm{dec}$ and a magnitude contribution of $+\alpha 20 \mathrm{~dB} / \mathrm{dec}$. The resulting controller yields a behavior as presented in Fig. $2 \mathrm{~b}$ where the contribution of the controller zeros and poles is modified by the operators' real orders. 


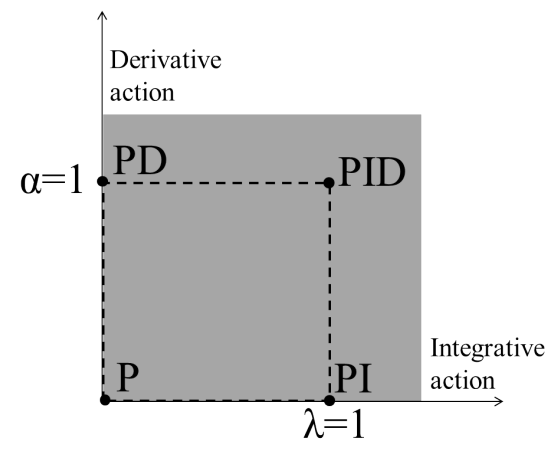

(a) Differentiation behavioral plane

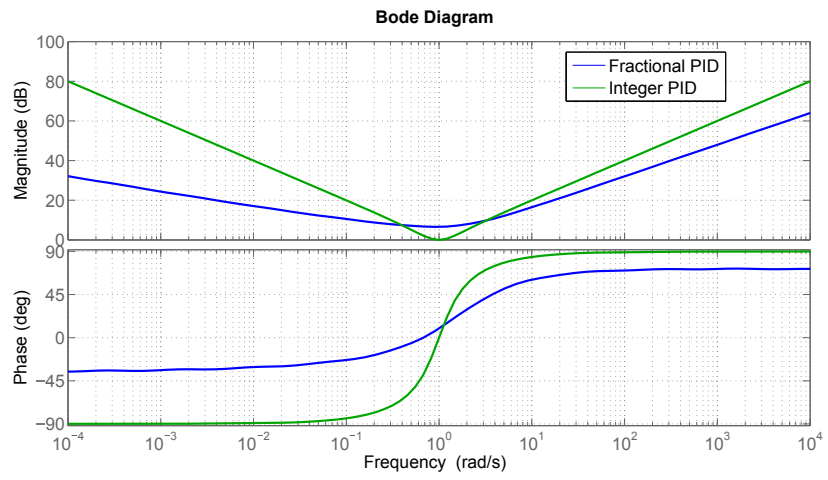

(b) Comparisson between a PID (green line) with the gains $(K p=K i=K d=1)$, and a Fractional PID with the same gains but the orders $\lambda=0.4$ and $\alpha=0.8$

Figure 2: Fractional-order control frequency effect illustration

\section{Vehicle Model}

This section introduces the experimental platform that will be later used in the real tests as well as the modelling of its longitudinal response.

\subsection{Cybercars}

These prototypes have been conceived as an efficient mobility service for urban environments, with the purpose of inserting the intelligent transportation systems into the cities and society [28]. They are equipped with on-board sensors and an embedded computer to process the gathered data, in order to generate the lateral and longitudinal commands. To provide car-following capabilities, Cybercars are equipped with front LiDAR (Light Detection and Ranging) sensors to measure the distance between the ego-vehicle and its preceding one. They are also capable to transmit their dynamic variables through communication links to the other string members for further processing. The experimental platform employed for this work is the 4-wheeled electrical prototypes Cycabs (see Fig. 3a). They were conceived to operate in urban scenarios with frequent interaction with vulnerable road users and for this reason their maximum speed is set to 5 $\mathrm{m} / \mathrm{s}$.

\subsection{Model Identification}

For the longitudinal model identification, a reference speed profile is given to the low level control of the vehicle. Fig. $3 \mathrm{~b}$ shows the experimental identification, where the black line describes the given reference speed, red line depicts the measured speed and blue one shows the fitted model response.

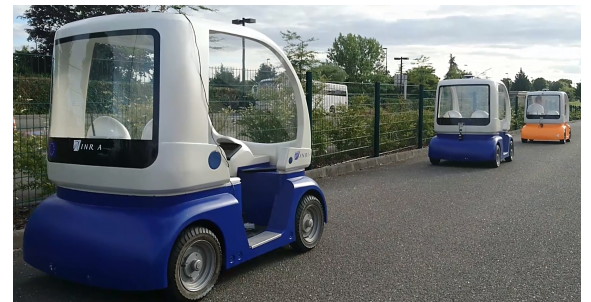

(a) Cycabs string formation

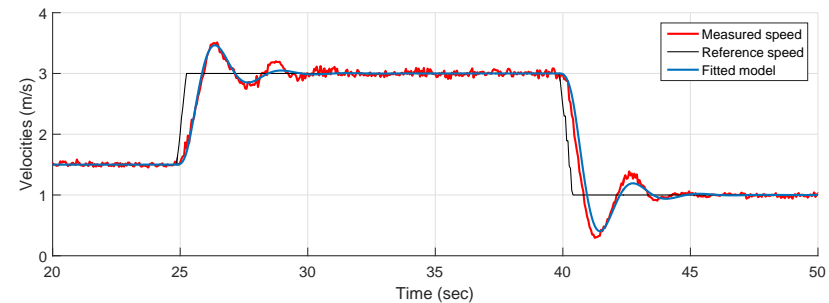

(b) Speed reference, vehicle real speed and model

Figure 3: Experimental platforms illustration and dynamic behavior modelling 
For the model identification, the MATLAB toolbox $i d e n t$ is used. The experimental identification process demonstrates that the longitudinal vehicle dynamics and actuator lags can be modelled using a second-order transfer function as:

$$
G p(s)=\frac{\omega_{n}^{2}}{\omega_{n}^{2}+2 \xi w_{n} s+s^{2}}
$$

where $\xi=0.3391$ is the damping factor and $w_{n}=2.5754 \mathrm{rad} / \mathrm{sec}$ is the plant natural frequency.

\section{ACC Control Design}

This section presents the control structure for the ACC algorithm and the fractional-order controller design.

\subsection{Control Structure}

For the ACC strategy, the controller output acts as a real-time correction over the ego-speed in the inner feedback loop. This generates the reference speed that the low-level control of the vehicle must track.

The outer feedback loop is composed by the $H(s)$ block, which describes the spacing policy $(H(s)=h s+1$, being $h$ the time gap). It provides the desired distance gap, that will be compared with the current measured distance to output the spacing error, upon which the control law $C(s)$ is further applied. The block $G p(s)$ represents the vehicle model identified in Sec. 4.

For the FOPD tuning process in the ACC case (see Fig. 4), the transfer function $G p_{f b}(s)$ summarizes the controlled model of the form:

$$
\frac{X_{i}(s)}{U_{i}(s)}=G p_{f b}(s)=\frac{G p(s)}{s(1-G p(s))}=\frac{\omega_{n}^{2}}{s^{2}\left(s+2 \xi \omega_{n}\right)} ;
$$

The block diagram in Fig. 4a shows the ACC control structure based on speed reference command. The inner control loop can be replaced by $G p_{f b}(s)$ leading to the control structure in Fig. $4 \mathrm{~b}$.

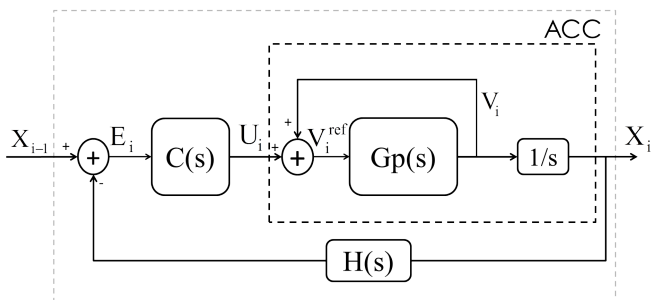

(a) ACC Control structure with $G p(s)$

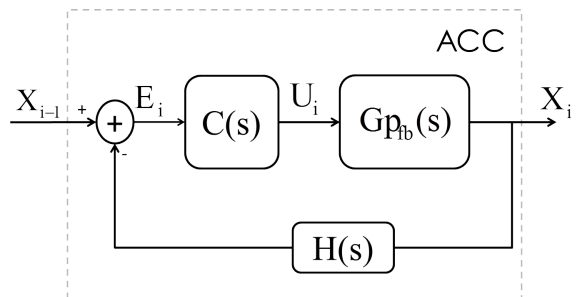

(b) ACC control structure with $G p_{f b}(s)$

Figure 4: ACC Control Structure

\subsection{FOPD Design Algorithm}

As it was previously stated, several state-of-the-art approaches have used lead controllers as PD instead of PID because the system damping and enhanced stability are fundamental in car-following approaches 20, 29]. It is proposed then to employ a non-integer-order PD controller for the gap regulation task in ACC, in other words, a Fractional-Order PD (FOPD) controller which control law is:

$$
\frac{U_{i}(j \omega)}{E_{i}(j \omega)}=C(j \omega)=K p \cdot\left(1+j \frac{\omega^{\alpha}}{\omega_{c}}\right)
$$

It has been demonstrated that for some applications, the optimal FOPD outperforms the optimal integerorder PD (IOPD) 4]. This type of controller posses a non-integer derivative, which allows to have one more parameter than IOPD to tune, and satisfy a more demanding frequency response. 
The FOPD controller design procedure is aimed not only to ensure the loop stability for the gapregulation task with a desired system bandwidth and phase margin, but also to increase the string stability when applied on a homogeneous string of vehicles. To achieve so, the procedure demands to obtain the optimal configuration of the controller gain $K p$, central frequency $\omega_{c}$ and the differentiation order $\alpha$ to fulfil the three requirements: maintain the vehicle response bandwidth when closing the loop, a desired phase margin and guarantee the string stability for the lowest time gap possible.

The estimation of the system bandwidth and phase margin when applying the CTG policy is studied over the loop expression $L_{A C C}(s)=G p_{f b}(s) C(s) H(s)$. Its gaincross frequency $\omega_{g c}$ should be similar to the vehicle's one (it is proposed $\omega_{g c}=3.5 \pm 0.1 \mathrm{rad} / \mathrm{sec}$ ), lower values of $\omega_{g c}$ limit the system reaction speed and higher values may yield saturation and non-linearities consequently. A phase margin of $\phi=(60 \pm 1)^{\circ}$ is targeted so the system is robust and stable, but not overdamped so it loses output-to-reference convergence speed. The string stability requirement is analysed over the closed loop expression Eq. 2, which for the ACC structure in Fig. 4 results:

$$
\left\|\Gamma_{A C C}(s)\right\|_{\infty}=\left\|\frac{X_{i}(s)}{X_{i-1}(s)}\right\|_{\infty}=\left\|\frac{C(s) G p_{f b}(s)}{1+C(s) G p_{f b}(s) H(s)}\right\|_{\infty} \leq 1 ; i \geq 2
$$

To retrieve the controller parameters, an optimization algorithm is executed for different values of $h$ using the Matlab command f solve, which allows to solve multi-objective non-linear optimization problem. The function to minimize results from the design requirements and interpreted as follows:

$$
F(h)=W_{1} \cdot\left|\omega_{g c}-3.50\right|+W_{2} \cdot\left|\phi-\frac{\pi}{3}\right|+W_{3} \cdot\left(\left\|\Gamma_{A C C}(s)\right\|_{\infty}-1\right) ;
$$

where $F(h)$ is the function to be minimized for a given time gap $h$. The general guideline to set the weights $W_{1}, W_{2}$ and $W_{3}$ is to define a considerably higher cost to the string stability condition, given that it is strictly required that $\left\|\Gamma_{A C C}(s)\right\|_{\infty} \leq 1$.

The algorithm is executed lowering the time gap until a Pareto-front is reached and one or more design requirements are no longer fulfilled due to the more demanding performance necessary to hold shorter time gaps. If a lower time gap is desired with string stable performance, one of the other design requirements must be relaxed. For the purpose of this work, the controller parameters that permits the lowest $h$ satisfying the design requirements stated above is kept. The expression:

$$
C(j \omega)=2.079 \cdot\left(1+j \frac{\omega^{1.075}}{2.640}\right)
$$

has been obtained as the optimal controller for the proposed requirements and the vehicle dynamics described by Eq. 7. With a time gap of $h=0.536 \mathrm{~s}$, it ensures a control structure with $\left\|\Gamma_{A C C}(s)\right\|_{\infty}=1$ and a phase margin of $\phi=59.148^{\circ}$ at the gaincross frequency $\omega_{g c}=3.556 \mathrm{rad} / \mathrm{sec}$.

\section{CACC Control Design}

This section introduces the control structure modifications to benefit from V2V information coming from the preceding vehicle. Then, the fractional-order control algorithm to deal with CACC system is presented.

\subsection{Control Structure}

The experimental platforms are commanded through a reference velocity, for this reason it is sent through the V2V communication link to the next vehicle in the string for its further usage as feedforward term. This approach is based on the inverse-model feedforward control under the assumption of homogeneous strings, permitting faster responses towards a speed change of the preceding vehicle without requiring the feedback loop to correct it. The proposed feedforward/feedback control structure has been also employed on several previous approaches, where good results have been obtained when applied in CACC [20, 24, 25]. Fig. 5 depicts that the plant input results from the addition of the feedforward term and the feedback FOPD controller output. 


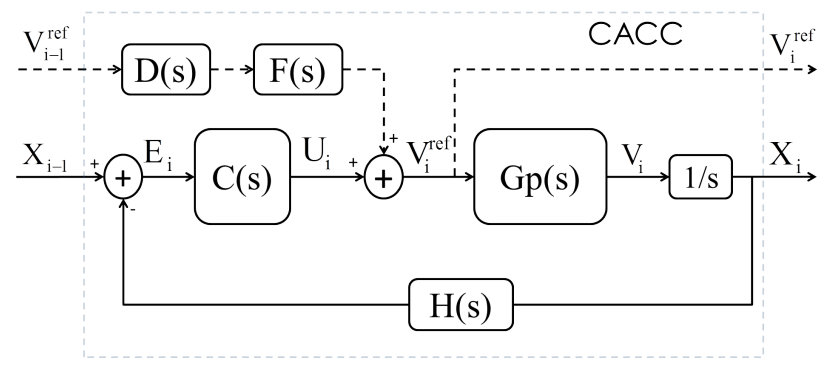

Figure 5: CACC control structure time gap possible that allows string stability.

\section{Comparative Study}

The term $F(s)$ is implemented to perform as a low-pass filter over the preceding vehicle reference speed to guarantee a smoother behavior. Furthermore, if $F(s)=\frac{1}{H(s)}$, the theoretical string stability is ensured for every time gap $h>0$ if ideal communications are considered with no delay [23]. Ideally, the employment of preceding vehicle reference speed guarantees string stability, but since $\mathrm{V} 2 \mathrm{~V}$ communication links present temporal delays (due to latency, channel congestion or encoding/decoding processing time), the feedback controller $C(s)$ is required to ensure the spacing error tracking as well as the loop robustness. This time delay is represented in the transfer function $D(s)=e^{-\theta s}$, where $\theta$ is the temporal delay in seconds. The string stability is studied with the following mathematical expression:

$$
\left\|\Gamma_{C A C C}(s)\right\|_{\infty}=\left\|\frac{X_{i}(s)}{X_{i-1}(s)}\right\|_{\infty}=\left\|\frac{D(s) F(s)+G p(s) C(s)}{s+G p(s) C(s) H(s)}\right\|_{\infty} \leq 1 ; i \geq 2
$$

The controller design requirements are the same stated above in Sec. 5 for the ACC structure. Since the communication delay directly affects the closed loop performance, a worst temporal delay of $\theta=0.08 \mathrm{sec}$ (measured in the experimental V2V network) is considered for the closed loop performance study in the parameter optimization algorithm. Since the inner feedback loop employed in ACC has been replaced with the feedforward term, the loop transfer function changes to $L_{C A C C}(s)=G p(s) G c(s) H(s) / s$.

The multi-objective optimization algorithm is employed to determine the controller parameters that satisfy: a loop bandwidth similar to the vehicle's $\left(\omega_{g c}=3.50 \pm 0.1 \mathrm{rad} / \mathrm{sec}\right)$, a phase margin of $(60 \pm 1)^{\circ}$ and strict string stability $\left\|\Gamma_{C A C C}(s)\right\|_{\infty} \leq 1$. As for the ACC configuration, the time gap is lowered until a Pareto-front is reached. After executing several iterations, the resulting controller is:

$$
C(j \omega)=2.483 \cdot\left(1+j \frac{\omega^{1.188}}{3.625}\right)
$$

which yields a phase margin of $\phi=60.031^{\circ}$ at $\omega_{g c}=3.519 \mathrm{rad} / \mathrm{sec}$ and guarantees the string stability for the given communication delay at a time gap of $h=0.254 \mathrm{sec}$.

As it was previously stated, a literature review shows several implementations using IOPD-based feedforward controllers for ACC/CACC systems [36, 20, 23, exhibiting good performance. A comparison with the FOPD controller proposed in this paper is carried out in this section. Since IOPD controllers account with two parameters, only two design requirements can be fulfilled. For this reason, two IOPD controllers are designed to do a proper comparison. The first controller is aimed to guarantee the loop bandwidth and its phase margin (referred as $I O P D^{P M}$ ), while the other should ensure loop bandwidth and maximize the string stability (referred as $I O P D^{S S}$ ). For both IOPD controllers, the solutions are sought for the lowest 


\section{1. $A C C$ and $C A C C$ Controllers}

In Tab. 1 the FOPD and the two IOPD controllers are shown for the ACC control structure, as well as the loop bandwidth, phase margin and minimum time gap of the system.

Table 1: Controller parameters obtained for ACC, minimum time gap allowed and stability metrics

\begin{tabular}{|c|c|c|c|c|c|c|c|}
\hline Controller & $K p$ & $\omega_{c}$ & $\alpha$ & $h[\mathrm{sec}]$ & $\phi\left[^{\circ}\right]$ & $\omega_{\text {gc }}[\mathrm{rad} / \mathrm{sec}]$ & $\|\Gamma(\mathrm{s})\|_{\infty}$ \\
\hline \hline FOP $D_{A C C}$ & 2.079 & 2.640 & 1.075 & 0.536 & 59.148 & 3.556 & 1.000 \\
$I O P D_{A C C}^{P M}$ & 1.613 & 2.015 & 1.000 & 0.572 & 60.078 & 3.505 & 1.000 \\
$I O P D_{A C C}^{S S}$ & 1.919 & 2.399 & 1.000 & 0.538 & 54.153 & 3.504 & 1.000 \\
\hline
\end{tabular}

All controllers produce the desired loop bandwidth, but it is observed that when designing an IOPD for the phase margin requirement, the time gap cannot be lowered as much as if the design requirement was maximizing string stability. On the other hand, the $F O P D_{A C C}$ controller is capable not only to ensure the string stability for the same time gap than $I O P D_{A C C}^{S S}$ does, but also to provide the desired phase margin. This demonstrates the compromise that exists when enhancing both the system performance and loop stability, which can be further improved profiting from the more flexible configuration of the fractionalorder controller.

Such behavior is depicted as well in Fig. 6. In the left figure the frequency response of $L_{A C C}(s)$ is showed when using the three controllers for their respective minimum time gap. The magnitude of their closed loop responses is illustrated in the right figure setting a $h=0.536$ sec. A clear difference is observed in the performance provided by the $I O P D_{A C C}^{P M}$ which results string unstable as the time gap set is lower than its limit. For the other controllers, the results showed in the table are visible in Fig. 6b since their closed loop performance results equal (a $\|\Gamma(s)\|_{\infty}=1$ ) for the same time gap, but the phase margin is reduced in the case of the $I O P D_{A C C}^{S S}$ (see Fig. 6a) with respect to what is provided by the $F O P D_{A C C}$ controller.

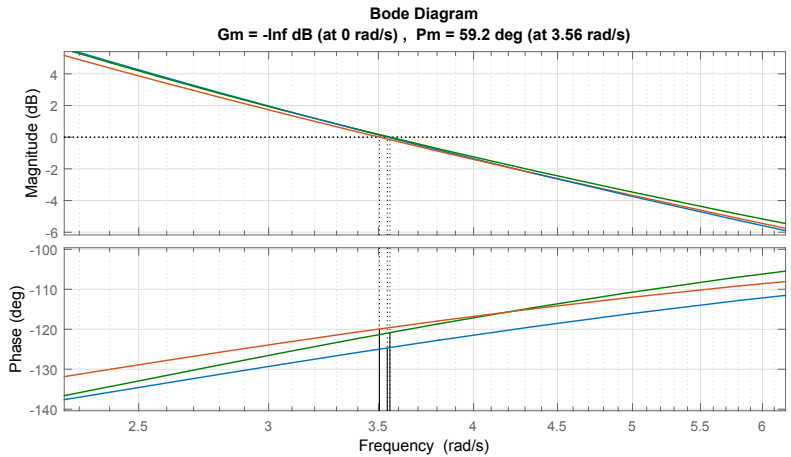

(a) Magnitude and phase of the loop response $L_{A C C}(s)$

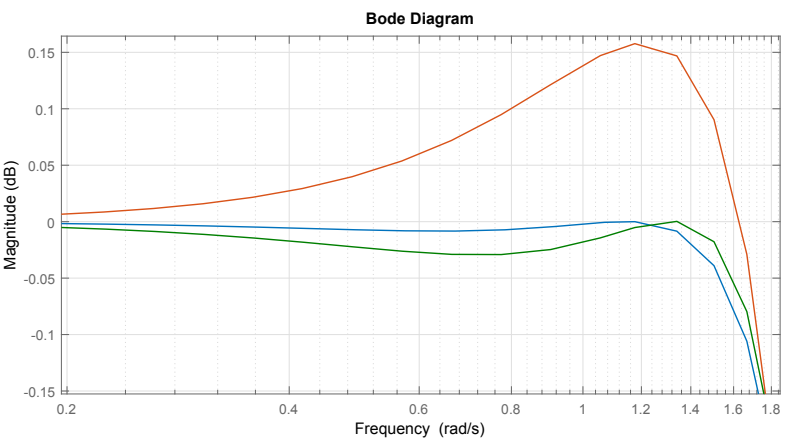

(b) Magnitude of the closed loop response if a time gap of $h=0.536 \mathrm{sec}$ is set

Figure 6: Frequency analysis of the control structure having as feedback controller: $F O P D_{A C C}$ (green line), IOPD ${ }_{A C C}$ (red line) and $I O P D_{A C C}^{P M}$ (blue line)

For the CACC scenario, the performance evaluation is also carried out with two IOPD controllers: the first one optimizes the string stability and the second one the loop phase margin. In Tab. 2 the resulting controllers are presented, with the stability performance that they provide when a $\mathrm{V} 2 \mathrm{~V}$ temporal delay of $\theta=0.08 \mathrm{sec}$ is considered.

It can be observed that if the $F O P D_{C A C C}$ attains the three objectives for a minimum time gap of $h=0.254 \mathrm{sec}$. On the other hand, when employing an IOPD controller for the same time gap, one must decide between either improving the system stability or increasing the closed loop performance with string 
Table 2: Controller parameters obtained for CACC, minimum time gap allowed and stability metrics

\begin{tabular}{|c|c|c|c|c|c|c|c|}
\hline Controller & $K p$ & $\omega_{c}$ & $\alpha$ & $h[\mathrm{sec}]$ & $\phi\left[^{\circ}\right]$ & $\omega_{\text {gc }}[\mathrm{rad} / \mathrm{sec}]$ & $\|\Gamma(\mathrm{s})\|_{\infty}$ \\
\hline \hline FOP $D_{C A C C}$ & 2.483 & 3.625 & 1.188 & 0.254 & 60.031 & 3.556 & 1.000 \\
$I O P D_{C A C C}^{P M}$ & 1.613 & 2.395 & 1.000 & 0.308 & 60.103 & 3.507 & 1.000 \\
$I O P D_{C A C C}^{S S}$ & 2.367 & 3.734 & 1.000 & 0.260 & 42.851 & 3.501 & 1.000 \\
\hline
\end{tabular}

stable behavior at a lower time gap. This is noticed in the properties of $I O P D_{C A C C}^{S S}$ controller, where even if it ensures string stability for almost the same time gap than the $F O P D_{C A C C}$, the resulting phase margin is compromised. If the desired phase margin must be guaranteed with an IOPD controller, the time gap exigence must be relaxed to provide a string stable behavior. This is illustrated in Fig. 7, where the left figure depicts the bode response of $L_{C A C C}(s)$ for the three mentioned controllers. In the right plot, the closed loop magnitude response is studied using each of the three controllers with a time gap of $h=0.254$ sec. In such figure, the $I O P D_{C A C C}^{P M}$ controller presents string unstable behavior. For the other two controllers the performances result almost similar, a difference is observed around $\omega=0.5 \mathrm{rad} / \mathrm{sec}$ where the closed loop magnitude yielded by the $I O P D_{C A C C}^{S S}$ results slightly higher than unity.

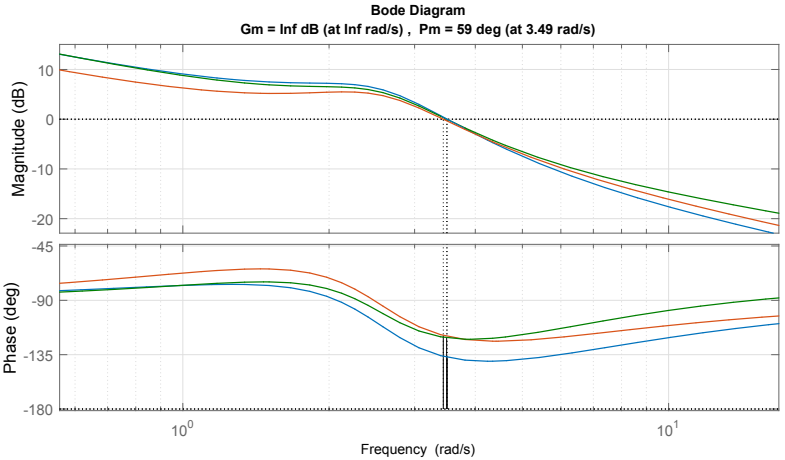

(a) Magnitude and phase of the loop response $L_{C A C C}(s)$

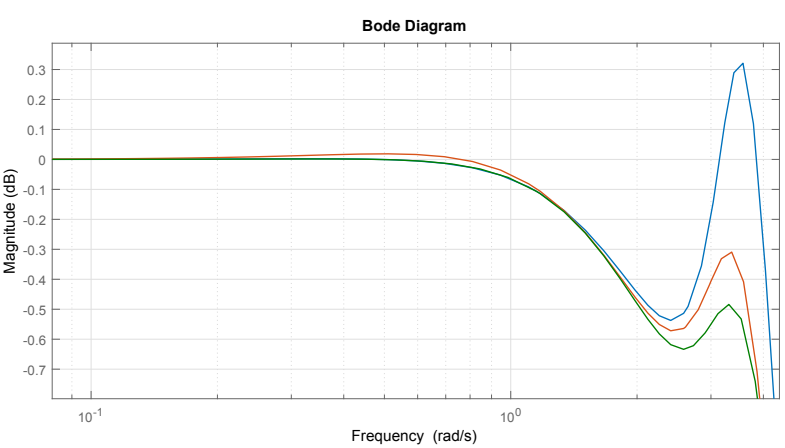

(b) Magnitude of the closed loop response if a time gap of $h=0.254 \mathrm{sec}$ is set

Figure 7: Frequency analysis of the control structure having as feedback controller: $F O P D_{C A C C}$ (green line), IOPDSSSCC (red line) and $I O P D_{C A C C}^{P M}$ (blue line)

Since the V2V temporal delay affects directly the string stability, the minimum time gap that can be set is modified. Having the three controllers presented in Tab. 2 the communication delay in $D(s)$ is varied as $\theta \in(0,0.3)$ sec to study how the time gap must be modified to maintain the string stability. Fig. 8 shows the study for the three controllers designed previously. It is observed that lower time gaps require low latency communications as would be expected. This evidences the relevance of the feedforward term in this type of control structures, where ideal communications provide string stability for all time gaps higher than zero [23. It is also worthy to mention that feedback loop robustness plays a key role for assuring string stability, overall when $\mathrm{V} 2 \mathrm{~V}$ temporal delays increase.

\subsubsection{Simulations}

A simulation of a string of 7 vehicles is carried out for both ACC and CACC algorithms (Fig. 9 and 10 respectively). Three scenarios are evaluated for each technique, an IOPD controller tuned to fix the loop bandwidth and phase margin, another IOPD configured to provide the desired bandwidth and to maximize the string stability and finally a FOPD that targets all the objectives stated before at the same time. For the sake of clarity, only the first and last vehicle performances are presented. The black solid line corresponds to 


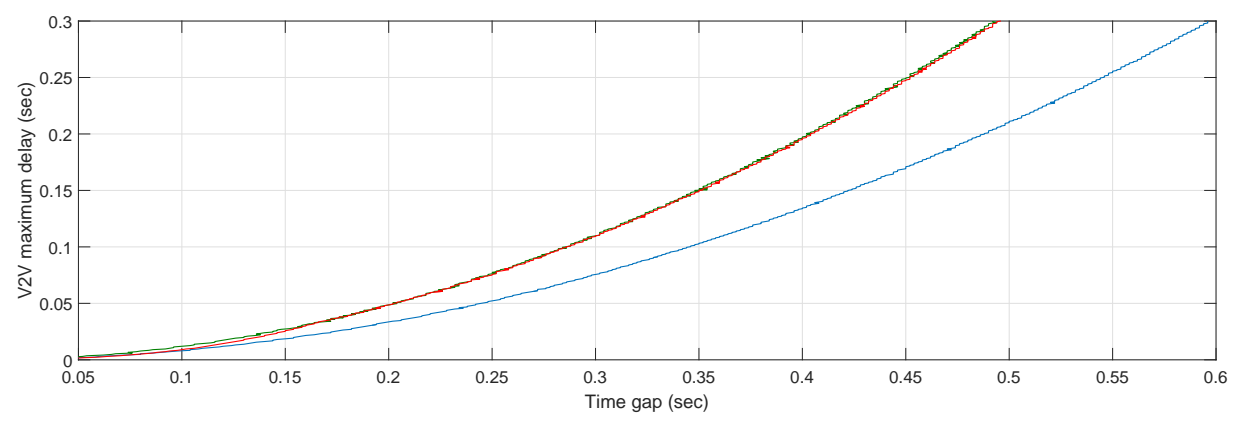

Figure 8: Maximum tolerable communication delay time for each time gap ensuring string stability for CACC strings for the $F O P D_{C A C C}$ (green line), IOPD $D_{C A C C}^{S S}$ (red line) and $I O P D_{C A C C}^{P M}$ controllers

the leader vehicle speed profile. This profile is identical in the three simulation to allow a fair comparison. The red, blue and green solid lines plots the responses of the 7 th $I O P D^{P M}, I O P D^{S S}$ and FOPD controlled vehicles respectively.

All variables are updated at the sample frequency of the vehicle odometry $f=100 \mathrm{~Hz}$. For the simulation purpose, the sensor noise has been omitted so the variables evolution is easier to visualize. For ACC and CACC scenarios, time gaps are set to $0.542 \mathrm{sec}$ and $0.254 \mathrm{sec}$ respectively added to a standstill distance of two meters. The same communication temporal delay of $\theta=0.08 \mathrm{sec}$ is assumed.
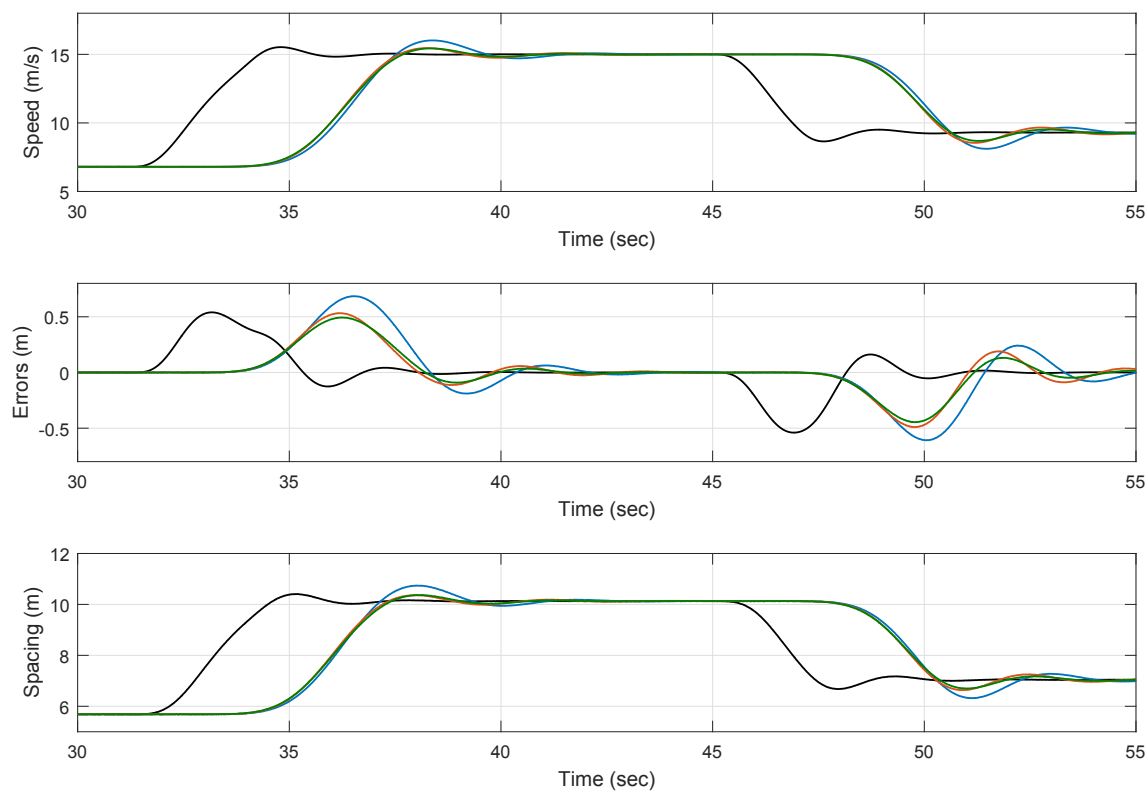

Figure 9: Simulation tests in ACC 7-vehicle strings using three different controllers: $I O P D_{A C C}^{S S}$ (red line), IOPD $P M C$ (blue line) and $F O P D_{A C C}$ (green line)

It is observed that with all the controllers, the speed changes are tracked and the spacing errors mitigated along time, showing stable performance. For CACC the inter-distances are smaller, and the speed changes are tracked in a faster way as a consequence of the lower time gap set. It can be highlighted that since the $I O P D_{A C C}^{P M}$ and $I O P D_{C A C C}^{P M}$ controllers are not designed to maximize the string stability, higher overshoots are observed. Such behavior is produced in both ACC and CACC and is overall visible in the spacing error evolution. One can appreciate that although $I O P D_{A C C}^{S S}$ and $I O P D_{C A C C}^{S S}$ controllers present the 

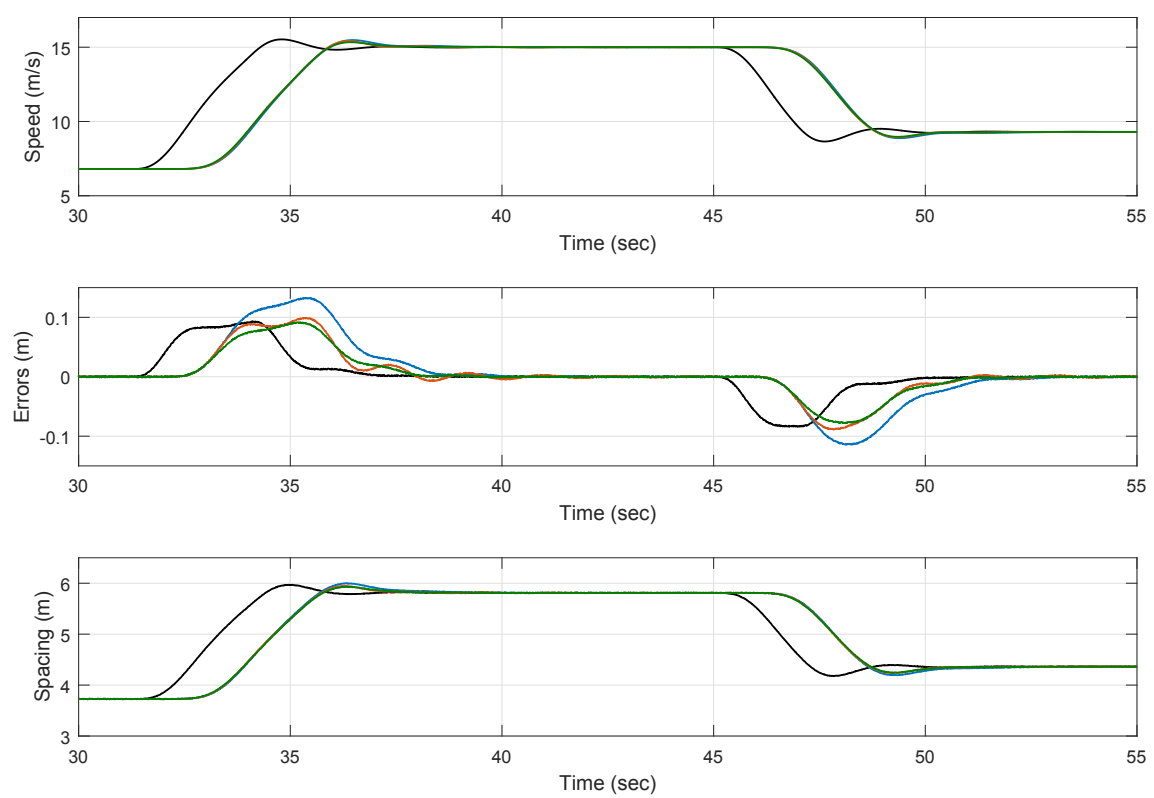

Figure 10: Simulation tests in CACC 7-vehicle strings using three different controllers: IOPD $D_{C A C C}^{S S}$ (red line), IOPD $P A C C$ (blue line) and $F O P D_{C A C C}$ (green line)

same attenuation towards speed changes than the fractional-order controllers, a more oscillating behavior is observed (more noticeable in the CACC scenario). These simulation results are in line with the theoretical values showed in Tab. 1 and 2 , where for the $I O P D^{S S}$ a lower phase margin is obtained.

\subsection{Real Platforms Implementation}

The designed controllers presented in Sec. 5 and 6 have been developed in the Laplace domain. For its implementation in real platforms architecture, the controller is discretized. The Tustin discretization method is selected due to its capability to translate continuous-time functions to the discrete domain easily. This method focuses on the translation of the differentiation operator $s^{\alpha}$ to a discrete finite order filter:

$$
s^{\alpha}=\left(\omega\left(z^{-1}\right)\right)^{\alpha}=\left(\frac{2}{T s}\right)^{\alpha} C F E\left\{\left(\frac{1-z^{-1}}{1+z^{-1}}\right)^{\alpha}\right\}
$$

where $T s$ is the sampling period of the discretization. After applying the discretization method, the function remains as a finite order rational discrete one. The Continued Fraction Expansion (CFE) method [33, 40] is used to approximate it to a " $n$ " finite order. Finally, the expansion results on a discrete-time transfer function as:

$$
s^{\alpha}=\left(\frac{2}{T s}\right)^{\alpha} \frac{\sum_{k=0}^{n} b_{k} z^{-k}}{1+\sum_{k=1}^{n} a_{k} z^{-k}}
$$

which coefficients $a_{k}$ and $b_{k}$ define the FIR discrete filter that results from the CFE. This manipulation is applied for all controllers with differential operators of fractional-order, both for ACC and CACC algorithms. As any discrete approximation of a continuous time function, the selected sample time must be short enough to provide a frequency response as desired. After studying the frequency response, a sample frequency of $20 \mathrm{~Hz}$ is used for the controller implementation as well as an order of $n=7$. It provides a good frequency approximation up to $50 \mathrm{rad} / \mathrm{sec}$, which is more than one decade above the closed loop bandwidth.

For the real tests, a 3-vehicle string (see Fig. 3a) is used. ACC and CACC scenarios are tested to compare the performances of the three controllers previously designed. For the sake of clarity, the graphics will show 
the leader speed (black line) and the speed of the third vehicle in the platoon for the three experiments. With respect to spacing errors and inter-distances, second and third vehicles' variables are depicted.

\subsubsection{Adaptive Cruise Control}

For the ACC technique, a distance corresponding to a time gap of $h=0.55 \mathrm{sec}$ is added to a standstill distance of three meters. The behavior observed in the simulations is also repeated in the real experiments.

The speed evolution results very similar for the three scenarios, except the $I O P D_{A C C}^{P M}$ controller which oscillations result slightly higher with respect to the others. Such behavior is more visible from the second to string stability limit that this controller yields. A smaller difference in the stabilization period is observed between the IOPD $D_{A C C}^{S S}$ and FOPD $D_{A C C}$ controllers performance due to the phase margin difference. The string stability results similar between both controllers since their limits are equal according to the theoretical analysis.
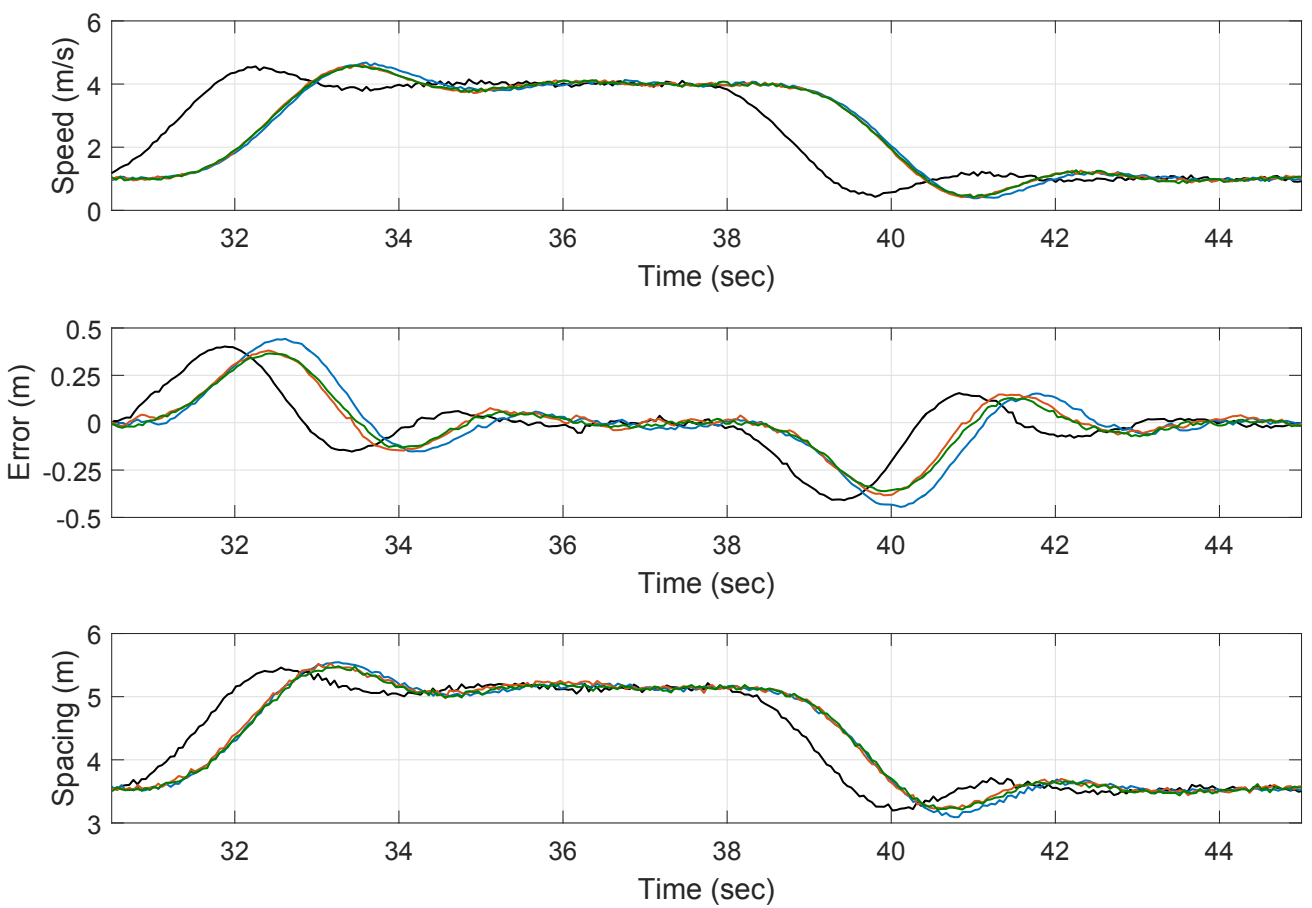

Figure 11: Speeds, errors and inter-distances for the ACC scenario, using the $I O P D_{A C C}^{P M}$ (blue line), IOPD ${ }_{A C C}($ red line) and $F O P D_{A C C}$ (green line) controllers

\subsubsection{Cooperative Adaptive Cruise Control}

The available communication links are now employed to perform the CACC tests. The three controllers in Tab. 2 are implemented following the proposed control structure CACC. As it was previously stated, measured communication delay is $0.08 \mathrm{sec}$. A time gap of $0.26 \mathrm{sec}$ is set for all vehicles in the string with a standstill distance of three meters.

Comparing to the ACC scenario, it is clear that shorter distances and faster gap-regulation maneuvers are achieved, which also leads to smaller peak errors. Even though the results are observed similar in terms of speed evolution, the spacing errors of the $F O P D_{C A C C}$ controller shows an enhanced stability with more attenuation upstream, while the $I O P D_{C A C C}^{S S}$ is at the stability limit and $I O P D_{C A C C}^{P M}$ shows an amplification of the spacing error. 

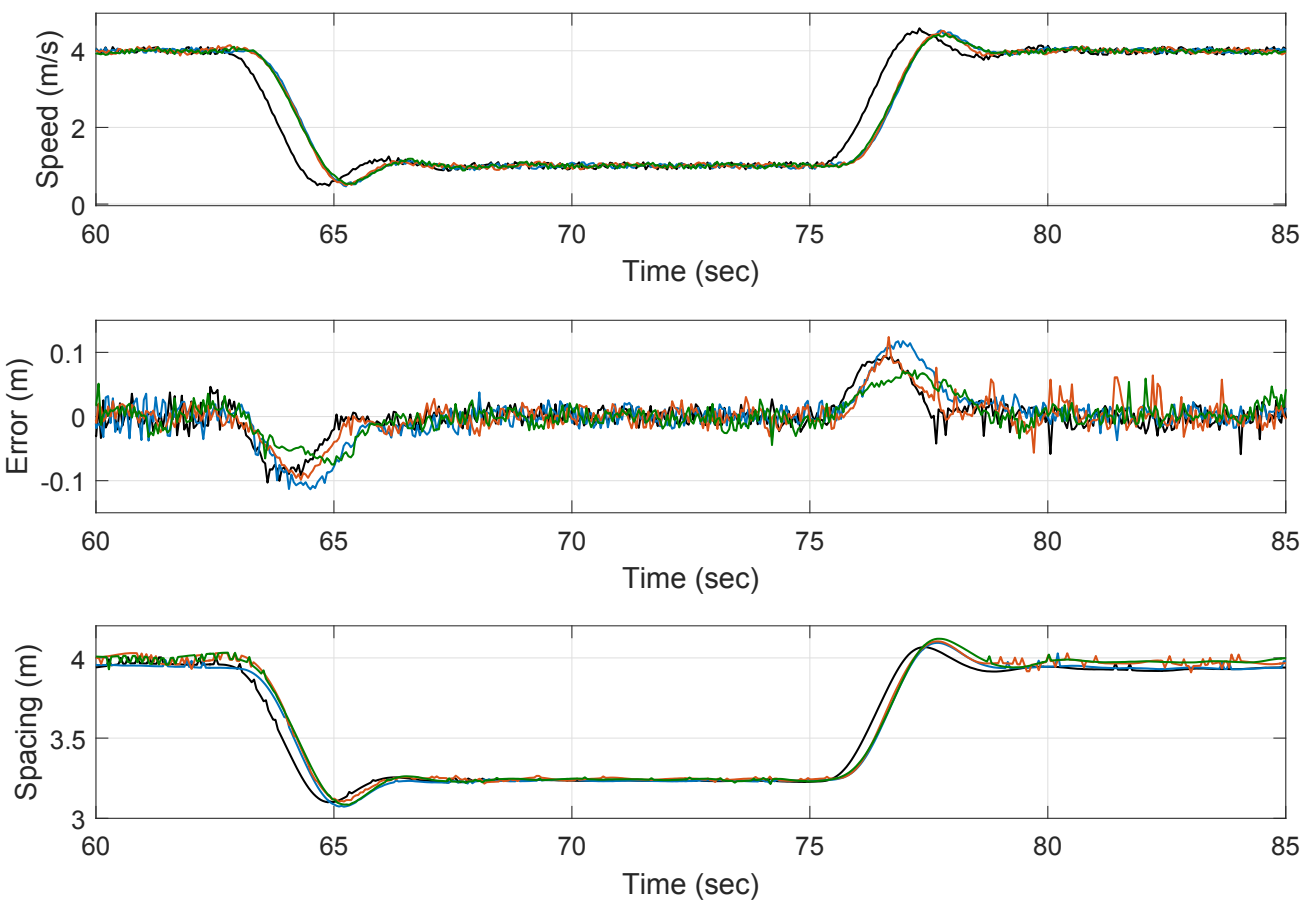

Figure 12: CACC state variables evolution in a 3-vehicle string with controllers: $I O P D_{C A C C}^{S S}$ (red line), IOPDP $P A C C$ (blue line) and $F O P D_{C A C C}$ (green line)

\section{Conclusions}

This paper presents the design, implementation and validation through simulation and real tests of fractional control algorithms for ACC and CACC techniques. Experiments are carried out using 3 Cybercars equipped with ranging sensors and $\mathrm{V} 2 \mathrm{~V}$ communication links, with the purpose of providing robust and string stable car-following in urban environments. A design algorithm oriented as a multi-objective optimization process is described for a fractional-order Proportional-Derivative controller, both for ACC and CACC. The more performing configuration of this controller permits to fulfil fundamental requirements as: loop bandwidth, robustness/stability through the phase margin and string stability maximization for the lowest time gap possible. It has been demonstrated that the fractional-order PD controller is able to optimize all criteria for a lower time gap than an integer-order PD controller would; without compromising any of the design requirements. This is confirmed by frequency response studies, and further demonstrated through simulations of an ACC/CACC string of 7 Cybercars. Real tests show the enhancement provided by a FOPD controller in terms of string stability over IOPD-based, when applied either in feedback (for ACC) or feedforward/feedback structures (for CACC). It can be also concluded that the more the gap-regulation task relies on the feedback loop (in the ACC case, or CACC with large communication delays), the selection of having a performing controller becomes more important. Future works will focus on the comparison of different control topologies for the CACC case.

\section{Acknowledgments}

This work has been founded by French Research Agency through the VALET project (ANR-15-CE220013). Authors express their gratitude to the RITS team for their support in the development of this work. 


\section{References}

[1] Åström, K.J., Hägglund, T., 2006. Advanced PID control. ISA-The Instrumentation, Systems, and Automation Society; Research Triangle Park, NC 27709.

[2] Caponetto, R., 2010. Fractional order systems: modeling and control applications. volume 72. World Scientific.

[3] Caputo, M., 1966. Linear models of dissipation whose Q is almost frequency independent. Annals of Geophysics 19, 383-393.

[4] Chen, Y., 2006. Ubiquitous fractional order controls? IFAC Proceedings Volumes 39, 481-492.

[5] Chen, Y., Petras, I., Xue, D., 2009. Fractional order control - a tutorial, in: Proc. American Control Conf, pp. $1397-1411$. doi $10.1109 /$ ACC. 2009.5160719 .

[6] El Ghouti, N., Serrarens, A., Van Sambeek, D., Ploeg, J., 2009. "connect \& drive" CACC for reducing congestion dynamics

[7] Flores, C., Milanés, V., Nashashibi, F., 2016. Using fractional calculus for cooperative car-following control, in: Proc. IEEE 19th Int. Conf. Intelligent Transportation Systems (ITSC), pp. 907-912. doi 10.1109/ITSC.2016.7795663

[8] Gao, F., Li, S.E., Zheng, Y., Kum, D., 2016. Robust control of heterogeneous vehicular platoon with uncertain dynamics and communication delay. IET Intelligent Transport Systems 10, 503-513.

[9] Geiger, A., Lauer, M., Moosmann, F., Ranft, B., Rapp, H., Stiller, C., Ziegler, J., 2012. Team annieWAY's entry to the 2011 grand cooperative driving challenge. IEEE Transactions on Intelligent Transportation Systems 13, 1008-1017. doi $10.1109 /$ TITS.2012.2189882

[10] Hosseinnia, S.H., Tejado, I., Milanés, V., Villagrá, J., Vinagre, B.M., 2014. Experimental application of hybrid fractionalorder adaptive cruise control at low speed. IEEE Transactions on Control Systems Technology 22, 2329-2336. doi 10. 1109/TCST.2014.2308837

[11] Hosseinnia, S.H., Tejado, I., Vinagre, B.M., Milanés, V., Villagrá, J., . ACC of a commercial vehicle using fractional order controllers for throttle and brake .

[12] Ioannou, P.A., Chien, C.C., 1993. Autonomous intelligent cruise control. IEEE Transactions on Vehicular Technology 42, 657-672. doi 10.1109/25.260745

[13] Li, H., Luo, Y., Chen, Y., 2010. A fractional order proportional and derivative (FOPD) motion controller: Tuning rule and experiments. IEEE Transactions on Control Systems Technology 18, 516-520. doi 10.1109/TCST.2009.2019120

[14] Liu, P., Ozguner, U., Zhang, Y., 2017. Distributed MPC for cooperative highway driving and energy-economy validation via microscopic simulations. Transportation Research Part C: Emerging Technologies 77, 80-95.

[15] Lu, X.Y., Hedrick, J.K., Drew, M., 2002. ACC/CACC control design, stability and robust performance, in: Proc. American Control Conf. (IEEE Cat. No.CH37301), pp. 4327-4332 vol.6. doi 10.1109/ACC.2002.1025325

[16] Luo, Y., Chen, Y., 2009. Fractional order [proportional derivative] controller for a class of fractional order systems. Automatica 45, 2446-2450.

[17] Lurie, B.J., 1994. Three-parameter tunable tilt-integral-derivative (TID) controller .

410 [18] Martinez, J.J., de Wit, C.C., 2007. A safe longitudinal control for adaptive cruise control and stop-and-go scenarios. IEEE Transactions on Control Systems Technology 15, 246-258. doi 10.1109/TCST.2006.886432

[19] Milanés, V., Shladover, S.E., 2014. Modeling cooperative and autonomous adaptive cruise control dynamic responses using experimental data. Transportation Research Part C: Emerging Technologies 48, 285-300.

[20] Milanés, V., Shladover, S.E., Spring, J., Nowakowski, C., Kawazoe, H., Nakamura, M., 2014. Cooperative adaptive cruise 15 control in real traffic situations. IEEE Transactions on Intelligent Transportation Systems 15, 296-305. doi 10.1109/TITS. 2013.2278494

[21] Monje, C.A., Chen, Y., Vinagre, B.M., Xue, D., Feliu-Batlle, V., 2010. Fractional-order systems and controls: fundamentals and applications. Springer Science \& Business Media.

[22] Narang, A., Shah, S.L., Chen, T., 2010. Tuning of fractional PI controllers for fractional order system models with and without time delays, in: Proc. American Control Conf, pp. 6674-6679. doi 10.1109/ACC.2010.5531353

[23] Naus, G., Vugts, R., Ploeg, J., v. d. Molengraft, R., Steinbuch, M., 2010a. Cooperative adaptive cruise control, design and experiments, in: Proc. American Control Conf, pp. 6145-6150. doi 10.1109/ACC.2010.5531596.

[24] Naus, G.J.L., Vugts, R.P.A., Ploeg, J., van de Molengraft, M.J.G., Steinbuch, M., 2010b. String-stable CACC design and experimental validation: A frequency-domain approach. IEEE Transactions on Vehicular Technology 59, 4268-4279. doi 10.1109/TVT.2010.2076320

[25] Nowakowski, C., O'Connell, J., Shladover, S.E., Cody, D., 2010. Cooperative adaptive cruise control: Driver acceptance of following gap settings less than one second, in: Proceedings of the Human Factors and Ergonomics Society Annual Meeting, SAGE Publications Sage CA: Los Angeles, CA. pp. 2033-2037.

[26] Oustaloup, A., Moreau, X., Nouillant, M., 1993. From the second generation CRONE control to the CRONE suspension, in: Systems, Man and Cybernetics, 1993.'Systems Engineering in the Service of Humans', Conference Proceedings., International Conference on, IEEE. pp. 143-148.

[27] Oustaloup, A., Sabatier, J., Moreau, X., 1998. From fractal robustness to the CRONE approach, in: Proc., ESAIM, pp. 177-192.

[28] Parent, M., Gallais, G., Alessandrini, A., Chanard, T., 2003. Cybercars: Review of first projects, in: Int. Conference on People Movers APM.

[29] Ploeg, J., 2014. Analysis and design of controllers for cooperative and automated driving .

[30] Ploeg, J., Scheepers, B.T.M., van Nunen, E., van de Wouw, N., Nijmeijer, H., 2011. Design and experimental evaluation of cooperative adaptive cruise control, in: Proc. 14th Int. IEEE Conf. Intelligent Transportation Systems (ITSC), pp. 260-265. doi 10.1109/ITSC.2011.6082981. 
[31] Ploeg, J., Shukla, D.P., van de Wouw, N., Nijmeijer, H., 2014. Controller synthesis for string stability of vehicle platoons. IEEE Transactions on Intelligent Transportation Systems 15, 854-865. doi 10.1109/TITS. 2013.2291493

[32] Podlubny, I., 1999. Fractional-order systems and pi/sup/spl lambda//d/sup/spl mu//-controllers. Automatic Control, IEEE Transactions on 44, 208-214.

[33] Podlubny, I., et al., 2004. Using continued fraction expansion to discretize fractional order derivatives. Nonlinear Dyn 38, $155-170$.

[34] Rajamani, R., Shladover, S., 2001. An experimental comparative study of autonomous and co-operative vehicle-follower control systems. Transportation Research Part C: Emerging Technologies 9, 15-31.

[35] Salvi, A., Santini, S., Valente, A.S., 2017. Design, analysis and performance evaluation of a third order distributed protocol for platooning in the presence of time-varying delays and switching topologies. Transportation Research Part C: Emerging Technologies 80, 360-383.

[36] Shaw, E., Hedrick, J.K., 2007. Controller design for string stable heterogeneous vehicle strings, in: Proc. 46th IEEE Conf. Decision and Control, pp. 2868-2875. doi 10.1109/CDC.2007.4435011.

[37] Shladover, S., Su, D., Lu, X.Y., 2012. Impacts of cooperative adaptive cruise control on freeway traffic flow. Transportation Research Record: Journal of the Transportation Research Board , 63-70.

455 [38] Shladover, S.E., Nowakowski, C., Lu, X.Y., Ferlis, R., 2015. Cooperative adaptive cruise control: Definitions and operating concepts. Transportation Research Record: Journal of the Transportation Research Board , 145-152.

[39] Shladover, S.E., Nowakowski, C., Lu, X.Y., Hoogendoorn, R., 2014. Using cooperative adaptive cruise control (CACC) to form high-performance vehicle streams .

[40] Song, B., Xu, L., Lu, X., 2014. A comparative study on tustin rule based discretization methods for fractional order differentiator, in: Information Science and Technology (ICIST), 2014 4th IEEE International Conference on, IEEE. pp. $515-518$.

[41] Swaroop, D., Hedrick, J.K., 1996. String stability of interconnected systems. IEEE Transactions on Automatic Control 41, 349-357. doi $10.1109 / 9.486636$.

[42] Swaroop, D., Huandra, R., 1998. Intelligent cruise control system design based on a traffic flow specification. Vehicle system dynamics 30, 319-344.

[43] Swaroop, D., Rajagopal, K.R., 2001. A review of constant time headway policy for automatic vehicle following, in: Proc. (Cat. No.01TH8585) 2001 IEEE Intelligent Transportation Systems ITSC 2001, pp. 65-69. doi 10.1109/ITSC.2001.948631

[44] Vinagre, B., Podlubny, I., Hernandez, A., Feliu, V., 2000. Some approximations of fractional order operators used in control theory and applications. Fractional calculus and applied analysis 3, 231-248.

470 [45] Vinagre, B.M., Monje, C.A., 2006. Introducción al control fraccionario. Revista Iberoamericana de Automática e Informática Industrial 3, 5-23.

[46] Vinagre Jara, B.M., Calderón Godoy, J., Suárez Marcelo, J.I., Monje Micharet, C.A., 2005. Teoría de control y cálculo fraccionario. Revista de la Real Academia de Ciencias Exactas, Físicas y Naturales 99, 241-258.

[47] Zhang, J., Ioannou, P., 2005. Adaptive vehicle following control system with variable time headways, in: Proc. 44th IEEE Conf. Decision and Control, pp. 3880-3885. doi 10.1109/CDC.2005.1582767

[48] Zhang, L., Orosz, G., 2016. Motif-based design for connected vehicle systems in presence of heterogeneous connectivity structures and time delays. IEEE Transactions on Intelligent Transportation Systems 17, 1638-1651.

[49] Zhao, J., Oya, M., Kamel, A.E., 2009. A safety spacing policy and its impact on highway traffic flow, in: Proc. IEEE Intelligent Vehicles Symp, pp. 960-965. doi 10.1109/IVS.2009.5164410 